\title{
Effective induction of oral anaphylaxis to ovalbumin in mice sensitized by feeding of the antigen with aid of oil emulsion and salicylate
}

\author{
Tomoko Shindo', Yukiko Kanazawa², Yoshiaki Saito', \\ Kohichi Kojima ${ }^{1}$, Motoyasu Ohsawa ${ }^{1}$ and Reiko Teshima ${ }^{3}$ \\ ${ }^{1}$ Hatano Research Institute, Food and Drug Safety Center, Ochi-ai, Hadano, Kanagawa 257-8523, Japan \\ ${ }^{2}$ Pharmaceuticals and Medical Devices Agency, Tokyo 100-0013, Japan \\ ${ }^{3}$ Division of Biochemistry and Immunochemistry, National Institute of Health Sciences, Setagaya, Tokyo 158-8501, \\ Japan
}

(Received November 25, 2011; Accepted December 27, 2011)

\begin{abstract}
It is important to evaluate the ability of novel proteins in food crops and products to elicit potentially harmful immunologic responses, including allergic hypersensitivity. We developed a novel mouse model of food allergy involving an oral challenge of a protein antigen after feeding of the antigen in combination with modulating factors often ingested in daily life, namely, dietary oil emulsion and salicylate. In the model, BALB/c mice were sensitized orally for three weeks with ovalbumin (OVA) in linoleic acid/lecithin emulsion, followed immediately by intraperitoneal injection of sodium salicylate. At the end of the sensitization, the incidence of mice positive for serum OVA-specific IgG1 but not IgE had significantly increased in the combined-sensitization group. After the 3-week sensitization, a single or double oral challenge with OVA effectively and significantly caused severe anaphylaxis, as compared with the groups sensitized with OVA in the emulsion or the vehicle alone. Moderate increase of plasma histamine and intestinal abnormality in histology was found only in the combined-sensitization group. Anaphylaxis symptoms in the sensitized mice were induced more by oral challenge than by intravenous challenge, suggesting a critical role for the mucosal system. This is the first model for successful induction of oral anaphylaxis in mice sensitized by feeding of food protein without adjuvant. It will be useful to elucidate the mechanism of food allergy and to detect modulating factors of oral allergy at sensitization using this model, which simulates real life conditions.
\end{abstract}

Key words: Anaphylaxis, Dietary oil emulsion, Salicylic acid, Food allergy, Mouse

\section{INTRODUCTION}

The potential allergenicity of novel proteins introduced into genetically engineered crops and that of proteins generated during the biotechnological process of food production is an important issue for the evaluation for safety of the food crops and products. Many approaches for assessing the allergenicity of such proteins have been proposed; these can be broadly classified into four areas, namely, the analysis of protein structure to predict allergenicity, serum screening of allergic proteins, new animal models, and proteomics (Selgrade et al., 2009; Ahuja et al., 2010).

The biochemical approaches for the assessment of allergenic proteins are grounded on comparisons of the serologic identity of the novel protein with human allergens, taking into account factors such as structural similarity, amino acid sequence homology, resistance to proteolytic digestion in a simulated gastric fluid, and immunochemical reactivity with serum of subjects with allergy (Ladics, 2008). Although such investigations provide important information, they do not directly assess the inherent sensitizing potential of proteins.

Animal models of food allergy are required for evaluating the sensitizing potential of allergenic proteins and to investigate mechanisms of food allergy. It is generally recognized that it is hard to reproduce food allergy in animals by oral feeding of a model allergenic protein at

Correspondence: Tomoko Shindo (E-mail: shindo.t@fdsc.or.jp) 
sensitization and challenge, mainly due to oral tolerance to the protein (Strobel and Mowat, 1998). Some mucosal adjuvants are known to abrogate oral tolerance to food proteins when fed with the protein antigen (Elson and Ealding, 1984; Snider et al., 1994; Clements et al., 1988; Gaboriau-Routhiau and Moreau, 1997). Consequently, in order to overcome oral tolerance, a number of mouse sensitization models of food allergy have been developed. Such models involve feeding of a protein antigen with a potent bacterial adjuvant (Li et al., 1999; van Wijik et al., 2005; Gizzarelli et al., 2006; Bowman and Selgrade 2008; Ganeshan et al., 2009), or alum (Diesner et al., 2008). In these models the production of antigen-specific $\mathrm{IgE}$ and/or IgG1 was enhanced after the sensitization. In a mouse model, feeding of food protein antigen together with cholera toxin has been shown to induce antigen-specific IgE and subsequently to elicit anaphylactic responses to oral challenge of the antigen (Li et al., 1999). Another mouse model using staphylococcus toxin has been reported to induce antigen-specific IgE and allergic symptoms by oral challenge of the antigen (Ganeshan et al., 2009). However, the emergence of food allergy associated with these bacterial toxins would be exceptional in a real living environment, and the mechanism by which the allergy occurs may differ from that in the case of common food allergy. It has thus been necessary to develop a mouse model of food allergy for sensitization by feeding of antigenic protein without pathogenic bacteria toxins.

Moreover, attempts have been made to create adjuvant-free sensitization mouse models. In a mouse model, antigen given orally, in excess and in isolation, was sufficient to induce antigen-specific IgE (Proust et al., 2008). Subsequently, anaphylaxis to the antigen was shown with intraperitoneal challenge, although oral challenge was not performed. Recently it has been reported that mouse models that employ dermal sensitization of dietary antigens without adjuvant can induce anaphylactic responses to an antigen given as an oral challenge (Hsieh et al., 2003; Birmingham et al., 2007; Gonipeta et al., 2009). However, these models are not relevant with respect to the route and doses of exposure to allergens at sensitization and/or elicitation of allergic symptoms. Therefore, we tried to develop a mouse model of food allergy that involved feeding of antigen without bacterial adjuvants at both sensitization and challenge.

Meanwhile, a number of dietary and/or environmental factors are known to affect the onset or exacerbation of food allergy. One suspected dietary factor relevant to food allergy is linoleic acid, a polyunsaturated fatty acid. The effect of dietary linoleic acid to exacerbate various allergic symptoms has been reported in experimen- tal and epidemiological studies (Sala-Vila et al., 2008; Miyake et al., 2011). A variety of oil emulsions have been used as an adjuvant in cosmetics, vaccines, therapeutics and foods. A dietary oil emulsion using soybean oil was reported to enhance immune responses to food antigens in mice (Kaneko et al., 2000). On the other hand, nonsteroidal anti-inflammatory drugs such as salicylic acid derivatives (Matsuo et al., 2005; Nakamura et al., 2006) and indomethacin (Louis et al., 1996) are known to promote the penetration of dietary proteins from the intestinal tract. Moreover, therapeutic use of salicylate in patients with food allergy was reported to aggravate their allergic condition, presumably by modulating food allergy (Shirai et al., 2003). Salicylate is metabolized mainly to salicylic acid in the body. Salicylic acid and its salts are now known to be natural components in some edible plants (Patterson et al., 2006), and also in animals where salicylate may primarily originate in endogenous benzoic acid (Patterson et al., 2008).

In light of the above considerations, we undertook development of a novel food allergy mouse model that employs combined feeding of the protein antigen and modulating factors often ingested in daily life, namely dietary oil emulsion and salicylic acid. In the novel model we succeeded in effective induction of oral sensitization with the food protein and subsequent anaphylactic elicitation by oral challenge of the antigen.

\section{MATERIALS AND METHODS}

\section{Reagents}

Ovalbumin (OVA) was used for the sensitization and challenge experiments. OVA (grade III) was purchased from Sigma-Aldrich Com. (St. Louis, MO, USA). Linoleic acid, egg yolk lecithin and sodium salicylate were purchased from Wako Pure Chem. Ind., Ltd. (Osaka, Japan). OVA was dissolved in saline or oil emulsion (saline, linoleic acid and lecithin were mixed at 5:4:1 by volume). The OVA solutions were used as the antigen solution at sensitization and challenge.

\section{Animals}

Female BALB/c mice (6 weeks old) obtained from Charles River Japan Inc. (Kanagawa, Japan) were housed under SPF conditions, receiving standard chow (CE-2, CLEA Japan, Inc., Tokyo, Japan) and tap-water ad libitum. Mice aged seven weeks were divided into groups ( $\mathrm{n}=6$ to 7 per group) and used for experiments. All experiments were performed according to the guidelines for the care and use of laboratory animals in Hatano Research Institute, Food and Drug Safety Center. 
Effective induction of oral anaphylaxis to ovalbumin in mice

\section{Oral antigen sensitization}

In the sensitization process mice in each group were orally administered $0.1 \mathrm{ml}$ individual antigen solution containing $1 \mathrm{mg}$ of OVA. Where necessary, intraperitoneal administration of $0.1 \mathrm{ml}$ saline containing $0.2 \mathrm{mg}$ sodium salicylate (SA) was given immediately afterwards. Unprimed control mice were orally given $0.1 \mathrm{ml}$ vehicle solution, the oil emulsion or saline alone. Each solution was given orally to animals using a feeding needle, five times a week for three weeks. For OVA sensitization, mice were divided into six groups and given different test materials: OVA/S and OVA/E groups were given OVA in saline or oil emulsion, OVA/S+SA and OVA/ $\mathrm{E}+\mathrm{SA}$ groups were given the OVA with intraperitoneal $\mathrm{SA}$, and None/S and None/E groups (vehicle controls) were given each vehicle alone without OVA, respectively. No significant changes were observed among all groups of mice in body weight and food intake during the sensitization process. No clinical signs were observed in any of the mice over the three weeks at oral sensitization.

On the day following the last sensitization, blood samples were collected from each mouse for determination of OVA-specific antibodies (IgE and IgG1) and total IgE. Sera prepared from the blood were kept at $-80^{\circ} \mathrm{C}$ until determination.

\section{Oral antigen challenge}

All groups of mice were orally challenged once or twice with OVA in saline or the oil emulsion corresponding to the vehicle at sensitization according to the protocol as follows: a) The one-time challenge: the day after the last sensitization OVA was orally given to mice with a feeding needle at a dose of $100 \mathrm{mg}$ in $0.4 \mathrm{ml}$ of the same vehicle as at the sensitization, S or E. b) The two-time challenge: one day after the first challenge mentioned in (a), a second oral challenge was performed with $75 \mathrm{mg}$ of OVA in $0.3 \mathrm{ml}$ individual vehicle.

To compare the different routes of the antigen challenge, in a separate experiment the second OVA was challenged orally or intravenously (i.v.) as in protocol (b). The i.v. challenge was performed by injection with $0.2 \mathrm{mg}$ of OVA in $0.2 \mathrm{ml}$ saline one day after the first oral challenge. The i.v. challenge dose was used to give a serum concentration of OVA comparable to that determined in mice orally given $75 \mathrm{mg}$ OVA.

\section{Measurement of OVA-specific antibodies in mouse sera}

To check the sensitization status, serum OVA-specific antibodies were determined on blood samples collected from mice the day after the last sensitization. The OVA- specific $\operatorname{IgE}$ and $\operatorname{IgG} 1$ titers in sera were determined by enzyme-linked immunosorbent assay (ELISA) (Alizadeh et al., 2006). Briefly, the ELISA was carried out with microtiter plates coated with $10 \mu \mathrm{g} / \mathrm{ml}$ of OVA solution. After blocking with bovine serum albumin (BSA), serial dilutions of mouse serum in phosphate buffered saline (PBS) containing 1\% BSA were added to each well of the plate. After $2 \mathrm{hr}$ incubation, OVA-specific antibodies captured onto each well were detected using horseradish peroxidase-conjugated rat anti-mouse IgE (monoclonal)or goat anti-mouse IgG1-antibody (Bethyl Laboratories, Inc., Montgomery, AL, USA). For detection, the ELISA plate bound peroxidase was incubated with TMB (tetramethylbenzidine) and the enzyme reaction was stopped with phosphoric acid. Enzyme activity in each well was measured by absorbance at $450 \mathrm{~nm}$ with an automated microplate reader. OVA-specific IgE and IgG1 titers were calculated based on optical density of sample serum as usual.

The OVA-specific anaphylactic antibody (mainly IgE) levels in sera were also measured by passive cutaneous anaphylaxis (PCA) testing (Mota et al., 1968), using seven week-old male Sprague-Dawley rats (Charles River Japan Inc.). Additionally, total IgE concentrations were determined using an ELISA kit (YAMASA Co., Chiba, Japan) according to instructions by the manufacturer.

\section{Assessment of anaphylactic responses}

Anaphylactic responses to the antigen challenge were evaluated by scoring symptoms in the $30 \mathrm{~min}$ after the final challenge (Birmingham et al., 2007; Gonipeta et al., 2009). Scores were as follows: $0=$ no symptoms, $1=$ scratching and rubbing the nose and head, or ruffling the fur, 2 = simultaneous expression of scratching and ruffling the fur, 3 = nausea and diarrhea, $4=$ wheezing, labored respiration, and cyanosis of the mouth and the tail, $5=$ serious symptoms leading to death within $24 \mathrm{hr}$.

To measure plasma histamine levels, $30 \mathrm{~min}$ after the one-time challenge (protocol a), mouse blood was collected into chilled tubes containing disodium-EDTA. Aliquots of plasma obtained from the blood were kept frozen at $-80^{\circ} \mathrm{C}$ before determination. Plasma histamine levels were determined using an ELISA kit (MP Biomedicals, Irvine, CA, USA) according to instructions by the manufacturer.

\section{Histological evaluation}

Forty minutes after the second challenge with oral OVA (protocol b), mice were sacrificed for histological examination of small intestine after systemic anaphylaxis. A part of the jejunum and the ileum, respectively, was fixed 
in $0.1 \mathrm{M}$ phosphate-buffered $10 \%$ formalin $(\mathrm{pH} 7.2)$ and embedded in paraffin. Tissues were stained with hematoxylin and eosin (HE) and microscopically checked.

\section{Statistical analysis}

Statistical differences were analyzed on GraphPad Prism 5 software (Alexa, SanDiego, CA, USA), using Fisher's exact test for serum antibody titers, one-way ANOVA with Dunnett's multiple comparison test for total IgE and plasma histamine levels after logarithmic normalization, and Kruskal-Wallis test with Dunn's multiple comparison test for anaphylaxis scores.

\section{RESULT}

\section{Inducibility of OVA-specific antibodies in serum by oral sensitization of OVA in combination with oil emulsion and salicylate}

The sensitization status seen with oral administration of a food protein was examined based on the inducibility of the antigen-specific antibodies in sera of mice given OVA. OVA-specific IgE antibody in serum was not detectable as titers below 10 in all groups (Table 1). No significant change in total IgE level in serum was detected in any group (Fig. 1), and no positive response in PCA testing was shown for tested sera in any group (data not shown).

In contrast to IgE, OVA specific IgG1 was found to be inducible at sensitization. Anti-OVA IgG1 titer above 10 was defined as positive induction of specific IgG1, because a few sera of mice without OVA feeding showed titer 10 . Table 1 shows the incidence of positive mice with serum titers above 10 in anti-OVA IgG1 induction on the day after the last sensitization. In spite of OVA feeding, there were no positive mice for induction of the anti-OVA IgG1 in OVA/S and OVA/S+SA groups, as justly in the None/S and None/E groups without OVA sensitization. Some mice in the OVA/E group showed induction of serum OVA-specific IgG1, but the incidence of mice positive for the antibody induction was not significantly different between the OVA/E and the above OVA groups. In contrast, when mice were orally given OVA in oil emulsion followed by SA (OVA/E+SA group), positive mice in OVA-specific IgG1 induction increased with a wide range of titers from $10^{2}$ to $10^{5}$. The incidence of the positive mice was significantly higher in the OVA/ $\mathrm{E}+\mathrm{SA}$ group than in None/E group.

\section{Induction of anaphylaxis by oral challenge of OVA}

After the oral sensitization with respective solutions, the mice were challenged once by oral administration of OVA in S or E vehicle, and their anaphylactic responses were observed. Manifestations of systemic anaphylaxis after the challenge were scored as in Fig. 2. There was little or no anaphylactic response in all mice in the None/S and None/E vehicle groups, while some mice in groups

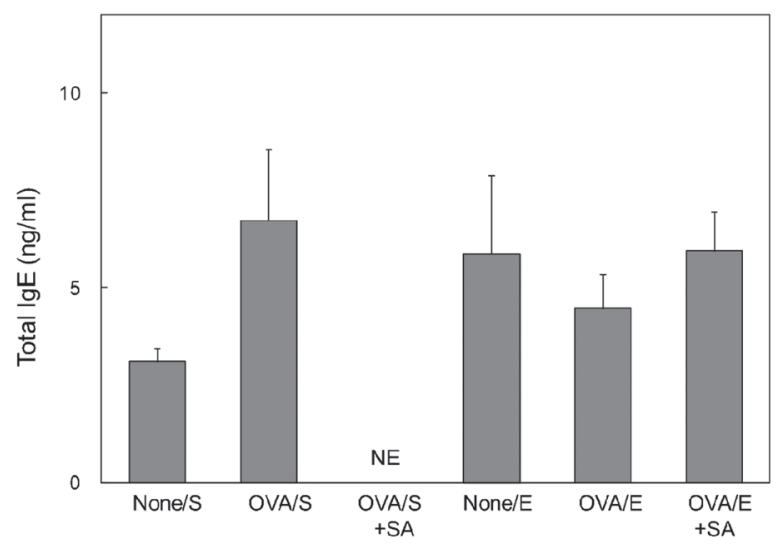

Fig. 1. Total IgE concentrations in sensitized mice. Sera from each group of mice were obtained the day after the last sensitization. Mice were orally sensitized with OVA in saline (OVA/S), OVA in saline plus salicylate (OVA/ $\mathrm{S}+\mathrm{SA})$, OVA in oil emulsion (OVA/E), or OVA in oil emulsion plus salicylate (OVA/E+SA), respectively. Control mice were given saline (None/S) or oil emulsion (None/E) alone. Total IgE concentrations were determined by ELISA. Bars show the average concentrations in each group. Data are expressed as mean \pm S.E. $(n=6)$. NE means not examined.

Table 1. Induction of anti-OVA IgE and IgG1 in BALB/c mice with different feedings

\begin{tabular}{|c|c|c|c|c|c|c|}
\hline \multirow[b]{2}{*}{ Antibodies } & \multicolumn{6}{|c|}{ Feeding Groups } \\
\hline & None/S & $\mathrm{OVA} / \mathrm{S}$ & $\begin{array}{c}\mathrm{OVA} / \mathrm{S} \\
+\mathrm{SA}\end{array}$ & None/E & $\mathrm{OVA} / \mathrm{E}$ & $\begin{array}{c}\mathrm{OVA} / \mathrm{E} \\
+\mathrm{SA}\end{array}$ \\
\hline $\operatorname{IgE}$ & $0 / 6$ & $0 / 6$ & $0 / 6$ & $0 / 6$ & $0 / 6$ & $0 / 6$ \\
\hline IgG1 & $0 / 6$ & $0 / 6$ & $1 / 6$ & $0 / 6$ & $2 / 6$ & $4 / 6^{*}$ \\
\hline
\end{tabular}

Values represent the number of positive mice in specific antibody induction/number of mice tested. Mice were defined as positive in the antibody induction with serum antibody titers of above 10 for $\operatorname{IgE}$ and $\operatorname{IgG} 1$, respectively. ${ }^{*} \mathrm{P}<0.05$ (Fischer's exact test) vs. None/E group (emulsion vehicle controls). 
Effective induction of oral anaphylaxis to ovalbumin in mice

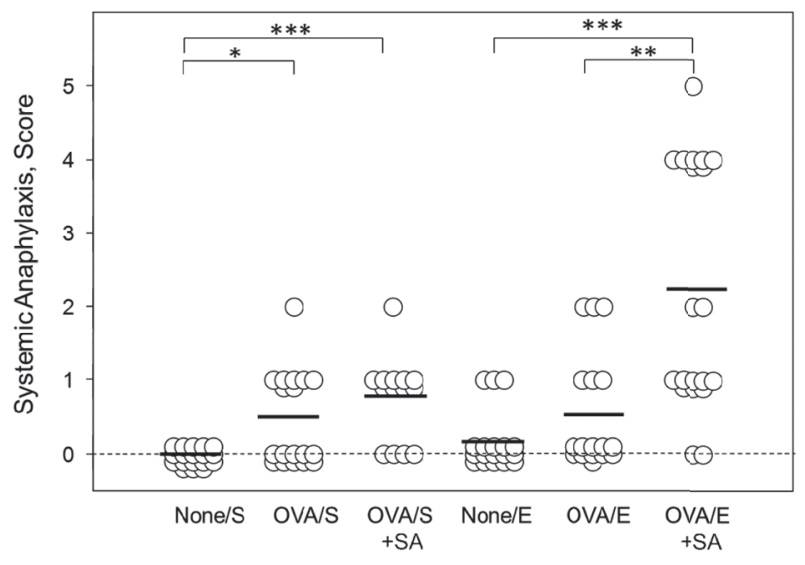

Fig. 2. Anaphylactic responses to oral OVA challenge of the OVA-sensitized mice. BALB/c mice were challenged with oral OVA (100 mg per mouse) on the day after the last sensitization. Anaphylactic symptoms within 30 minutes after the challenge were scored on a scale from 0 (no symptoms) to 5 (death within $24 \mathrm{hr}$ ), as described in Materials and Methods. Each symbol shows the greatest score of symptoms in each mouse. Bars represent the average score for each group ( $\mathrm{n}=$ 14 to 20). Statistical differences were analyzed among groups by Kruskal-Wallis test with Dunn's multiple comparison test. ${ }^{*} p<0.05,{ }^{* *} p<0.01,{ }^{* * *} p<0.001$.

sensitized with OVA alone (OVA/S and OVA/E groups) showed mild responses such as scratching and rubbing the nose and head or ruffling the fur. Similar response features were obtained also in OVA/S+SA group, although they were different in anaphylaxis scores from the None/S and None/E vehicle controls. In contrast, the OVA/E+SA group of mice experienced severe symptoms, although a few animals did not exhibit anaphylactic response. The symptoms included wheezing, labored respiration, and cyanosis at the mouth and the tail, and death within $24 \mathrm{hr}$. The responses in the OVA/E+SA group were more severe and significantly different from those in other emulsion vehicle groups (OVA/E and None/E), and likely different from those in the OVA/S+SA group. Moreover, the oil emulsion using soy lecithin instead of egg yolk lecithin was also examined, because anaphylaxis to OVA might be promoted by contaminants in egg lecithin. As a result, anaphylaxis was found to be induced in the OVA/E+SA group by soy lecithin as well as egg yolk lecithin (data not shown). These results indicate oral OVA challenge can effectively elicit anaphylactic response in mice sensitized with oral OVA in oil emulsion with then given SA.

As shown in Fig. 3, plasma histamine levels after OVA challenge were significantly higher in the OVA/E+SA and

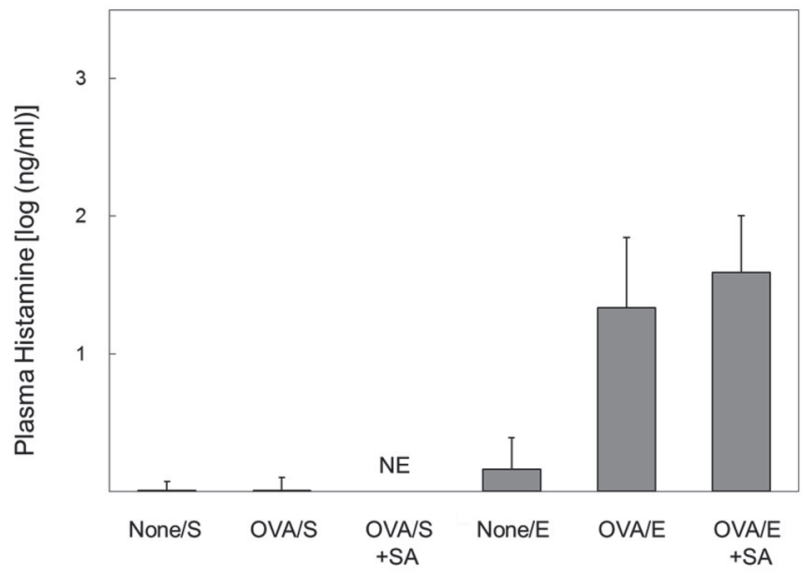

Fig. 3. Plasma histamine levels at the challenge. BALB/c mice were challenged orally with $100 \mathrm{mg}$ of OVA on the day after the last sensitization. Plasma histamine level was determined in blood collected from each mouse at 30 min after the challenge. Each column and vertical line represents the mean and S.E. (normalized as $\log$ concentration, $n=6$ ), respectively. NE means not examined. Statistical differences were analyzed between groups by Dunnett's multiple comparison tests.

OVA/E groups, as compared with the None/E group. A trace amount of histamine was found in the OVA/S group.

\section{Effect of the challenge route on the anaphylactic responses}

Next, in order to improve the inducibility of anaphylaxis in the OVA/E+SA group, the sensitized mice were challenged twice with OVA according to protocol (b). In the same way, to evaluate effectiveness of oral challenge on induction of anaphylaxis, the inducibility of anaphylactic responses was compared between mice challenged orally and i.v. at the second OVA.

The results in Fig. 4a show that all groups of mice challenged twice with oral OVA responded in a manner similar to the differential induction of anaphylaxis observed in mice challenged once (Fig. 2). Furthermore, all mice in the OVA/E+SA group challenged twice clearly manifested more severe symptoms than when challenged once. On the other hand, the i.v. challenged groups at the second OVA showed less responsiveness (Fig. 4b) even in the OVA/E+SA group, in spite of the i.v. challenge dose sufficient to provoke anaphylaxis and comparable in serum OVA concentration to the oral dose.

Histopathology of the intestinal tract in the twice orally challenged mice is shown in Fig. 5. Fig. 5a shows an image of intestinal tract from mice in $\mathrm{S}$ group (saline controls) given OVA challenges. The histological features did 
T. Shindo et al.
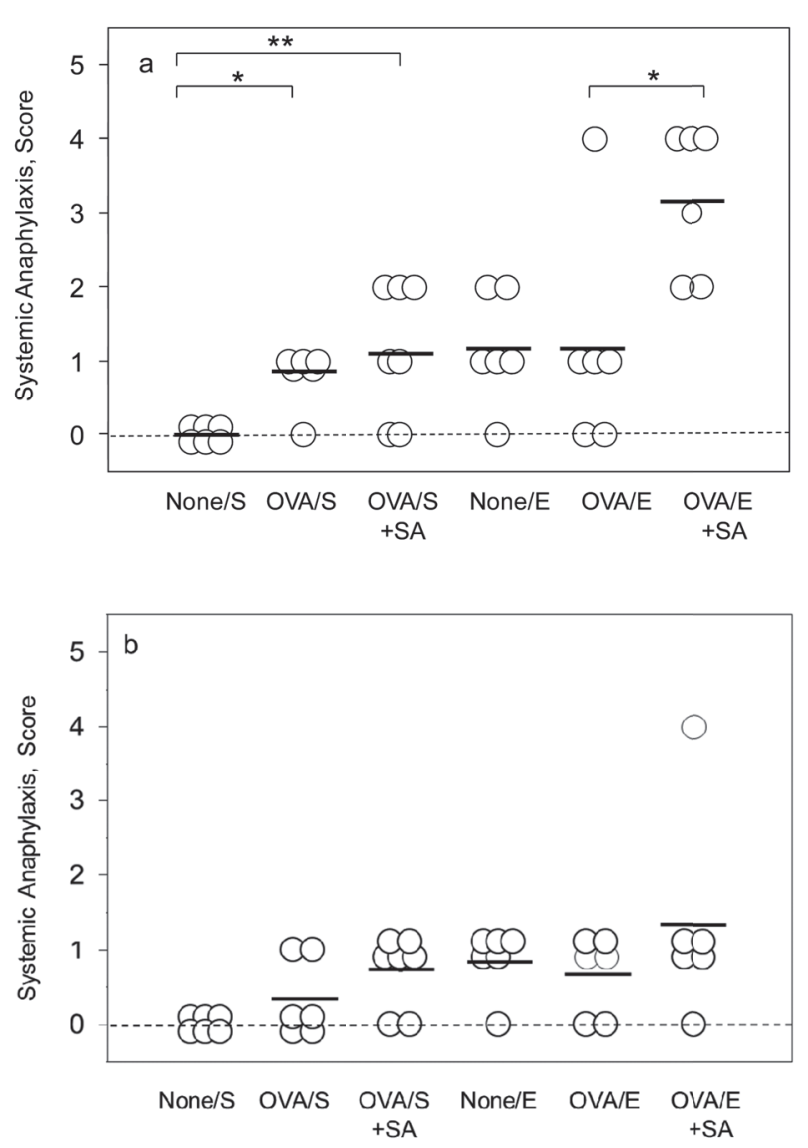

Fig. 4. Comparison of the different challenge routes on OVAinduction of systemic anaphylactic responses between (a) oral and (b) intravenous administration. On the day after the last sensitization, mice were challenged orally with $100 \mathrm{mg}$ of OVA. One day later, the mice were challenged orally (75 mg) or intravenously $(0.2 \mathrm{mg})$ with OVA. During the first $30 \mathrm{~min}$ after the challenge, the symptoms of anaphylaxis were scored as described in Materials and Methods. Each symbol shows the greatest score of symptoms in each mouse. Each bar represents the average score of each group. Statistical differences were analyzed among groups by Kruskal-Wallis test with Dunn's multiple comparison test.

not differ from the controls in all groups of mice except the OVA/E+SA group, while mice in the OVA/E+SA group gave abnormal findings on small intestine. The histological alterations were mild even under severe anaphylaxis, and appeared more frequently after the second challenge. Typical histopathological findings in the OVA/ $\mathrm{E}+\mathrm{SA}$ group included dilatation of the lumen in intestinal villi, together with accumulation of lymphocytes, a slight dilatation of capillary in the lamina propria, and shortening of the villus (Figs. 5b, $c$ and d). These findings were not found in other groups with/without OVA challenge.

\section{DISCUSSION}

Many mouse sensitization models of food allergy that use oral immunization with a protein antigen plus a potent adjuvant have been developed. Oral sensitization models without an adjuvant have also been proposed. One is the sensitization model using oral immunization with protein alone but at unusually high dose (Proust et al., 2008). The other is the transdermal immunization of protein antigen alone (Hsieh et al., 2003; Birmingham et al., 2007). These sensitization models, however, are not conventional at exposure to food antigen regarding the route, dose and co-existing factors. Thus, there exist few models that can evaluate the sensitizing potential of food allergens and explore how patients are sensitized in actual cases of food allergy. Moreover, there are no mouse models of food allergy elicited by oral exposure of antigen at both sensitization and challenge. The present research showed that in our mouse model using dietary oil emulsion and salicylate, mice were sensitized by oral feeding of OVA, an allergenic protein, and experienced anaphylactic responses to oral feeding of OVA. This mouse model demonstrates for the first time that anaphylactic food allergy can be induced by oral exposure to an allergen at both sensitization and challenge when simulating actual food allergy.

OVA is one of the major causes of food allergy in children with features of anaphylactic hypersensitivity. In our model the type 1 hypersensitivity responses to oral OVA were evidently exhibited in the OVA/E+SA groups. Typical anaphylactic symptoms appeared within 30 min after oral challenge and were associated with mild histological changes in small intestine and increase of plasma histamine. The strength of anaphylactic responses to OVA in this model was comparable to that in the mouse model using milk or peanut antigen with cholera toxin adjuvant (Li et al., 1999, 2000).

It is known that murine systemic anaphylaxis can be caused by two independent mechanisms, mediated by $\operatorname{IgE}$ or IgG antibodies (Strait et al., 2002; Finkelman 2007; Mukai et al., 2009). IgE antibodies play an important role in mediating type 1 hypersensitivity responses in humans. Systemic anaphylaxis has been generally recognized as severe allergic reactions caused by IgE-mediated activation of mast cells leading to release of chemical mediators including histamine. However, neither anti-OVA IgE and total IgE antibodies nor primarily IgE-dependent PCA reaction were detected in the novel model, although at the extended sensitization for four weeks OVA-specific 

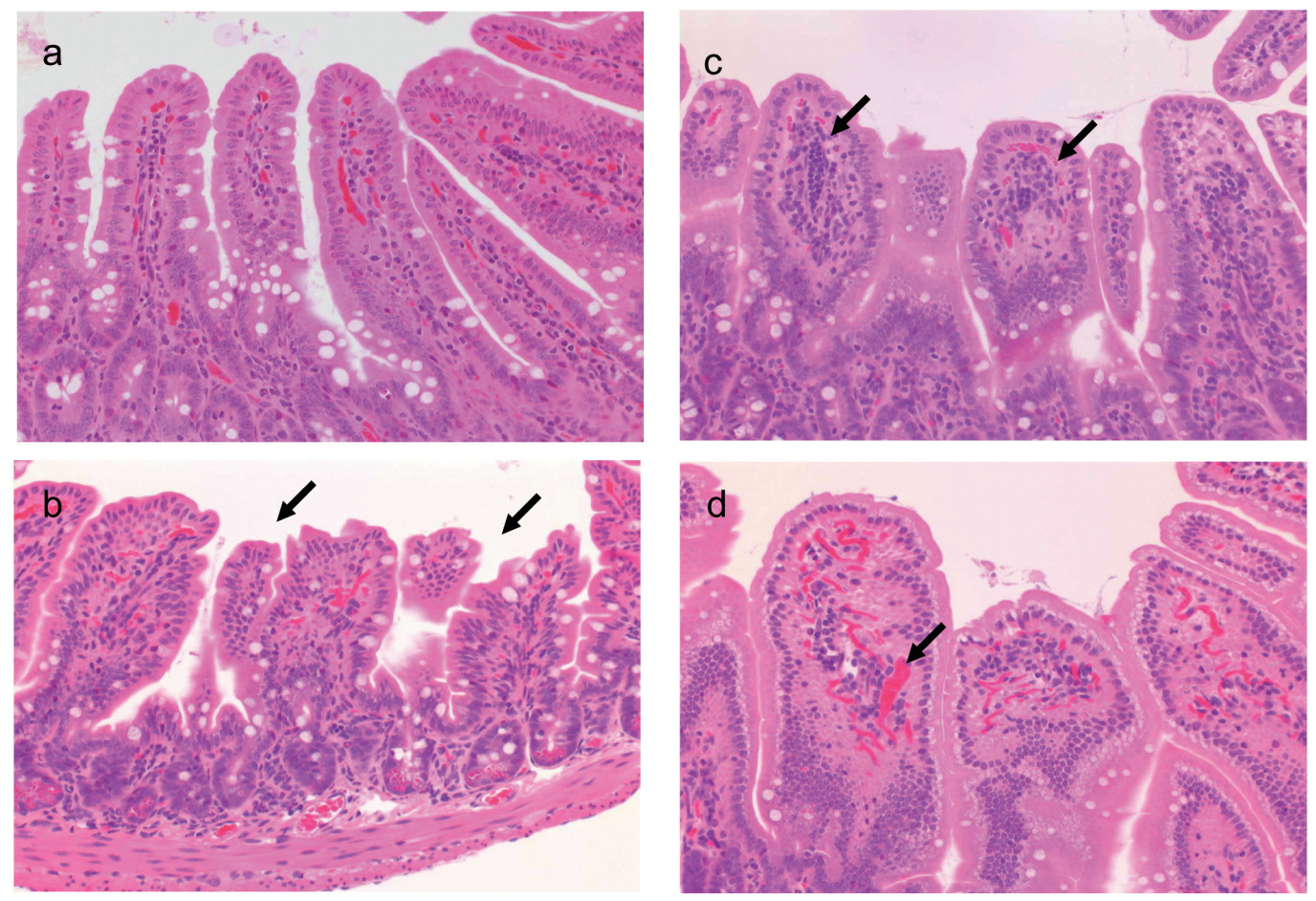

Fig. 5. Histological alterations in small intestine after the second OVA challenge. Histological images (magnification: $x$ 120) of hematoxilin-eosin stained small intestine are for None/S group (a) and OVA/E+SA group (b, c and d). Arrows in photos indicate typical images for shortening of the villus (b), accumulation of lymphocyte in the lamina propria (c) and dilatation of a capillary vessel in the lamina propria (d).

IgE became detectable in serum, followed by aggravation of anaphylactic responses (data not shown). On the other hand, anti-OVA IgG1 antibody was detected in serum of most of the OVA/E+SA mice with marked induction (Table 1). Interestingly it has been reported that although oral administration of allergenic hazelnut alone did not sensitize BALB/c mice, oral hazelnut with an antiulcer drug, omeprazole, sensitized mice with induction of hazelnut-specific IgG1 but not IgE, leading to immediate hypersensitive skin reactions (Schöll et al., 2005). In the OVA/E and OVA/E+SA groups, a similar increase of plasma histamine mediating IgE-dependent anaphylaxis was seen, but anaphylaxis was manifested more severely in the OVA/E+SA group than in the OVA/E group. The combination of oil emulsion and salicylate could contribute primarily to induce OVA-specific IgG1 which led to elicitation of anaphylaxis.

In mice, IgG1 antibodies are also known to mediate immediate hypersensitivity reactions (Oettgen $e t$ al., 1994; Lei et al., 1996; Miyajima et al., 1997). In our model mice, elevated levels of IgG1 were closely associated with the severity of systemic anaphylaxis (Table 1 and Fig. 2). The experimental findings indicate that the mice of the OVA/E+SA group were sensitized to induce OVA-specific IgG1 but not IgE. It suggests that the IgG1 was initially involved in provoking systemic anaphylaxis or that not IgE but IgG1 was dominantly induced at oral sensitization with antigenic protein in oil emulsion plus salicylate. Recently, basophils and probably macrophages, not mast cells, were shown to be the major players in IgG- but not IgE-mediated systemic anaphylaxis involving platelet-activating factor (PAF) (Tsujimura et al., 2008; Mukai et al., 2009). These mechanisms of anaphylaxis may also be relevant in humans, as serum PAF levels are correlated with the severity of anaphylaxis in patients (Vadas et al., 2008). Furthermore, a study of meta-analysis for patients with food allergy reported that although allergen-specific IgE in serum was highly relevant to allergic symptoms and useful diagnostic marker, a considerable part of patients did not always increase the specific IgE in serum (García et al., 2009). Taken together, the systemic anaphylaxis in the novel model of food allergy may be caused by an alternative pathway involving allergen-specific IgG1 antibody. The role of the IgE 
antibody in this model requires further research.

It is meaningful that oral challenge of OVA to the sensitized model mice caused more effective responses in frequency and intensity of anaphylactic symptoms when compared with i.v. challenge of the antigen, as both challenges were estimated to give roughly equivalent serum levels of OVA (Figs. 4a and 4b). The differential response by the antigen challenge routes may indicate a critical role for the gastrointestinal tract in provocation of the anaphylactic response in food allergy.

Oil emulsions are known to work as adjuvants. Soybean oil emulsion that did not affect oral tolerance was effective to provoke humoral immune response to B-lactoglobulin in mice, when administered orally together with the protein (Kaneko et al., 2000). The dietary oil emulsion used in our model may make OVA resistant to digestion by incorporating it, and/or contribute to enhance cellular penetration of the protein into gut, thus promoting antigen presentation.

SA may also contribute to increase the mucosal uptake of food protein from intestinal tract and to assist antigen presentation, thereby suppressing oral tolerance to the protein. Impairment of oral tolerance can be caused by a certain number of therapeutic agents (Kim and Ohsawa, 1995; Louis et al., 1996; Pecquet et al., 2004) and a dioxin (Chmill et al., 2010) and its implication in food allergy has been discussed. However, OVA levels in serum of mice were alike, irrespective of SA exposure (data not shown). Moreover, only in the case where SA and oil emulsion were combined as in the OVA/E+SA group was OVA notably effective in inducing allergic responses (Fig. 2). It suggests that the two factors costimulate induction of allergenic response to OVA. SA as well as aspirin prevents prostaglandin formation by inhibiting cyclooxygenases (COX). Recently it was shown that the COX-2 in mucosal dendritic cells may be involved in induction of oral tolerance to dietary antigen via mediating activation of regulatory T cells (Broere et al., 2009). It may suggest an effect of SA different from that of oil emulsion in the sensitization process. Further research on the novel model may provide us with a profound insight on mechanisms for food allergy.

In conclusion, we first developed a novel mouse model of food allergy to elicite anaphylactic responses by oral feeding of allergenic protein both at the sensitization and at the challenge. Mice in this model were orally given an allergen, OVA, in combination with oil emulsion and SA at the sensitization. The oil emulsion and SA caused anaphylactic responses to the orally given allergen, mainly in association with induction of antigen-specific IgG1 antibody at sensitization. This real life-simulating model is expected to prove useful to elucidate the mechanism of food allergy, in particular the non- $\operatorname{IgE}$ dependent mechanism, and to detect modulating factors of oral allergy at sensitization.

\section{ACKNOWLEDGMENTS}

This study was partly supported by a Grant-in-Aid from the Ministry of Health, Labor, and Welfare (H15Food-003).

\section{REFERENCES}

Ahuja, V., Quatchadze, M., Ahuja, V., Stelter, D., Albrecht, A. and Stahlmann, R. (2010): Evaluation of biotechnology-derived novel proteins for the risk of food-allergic potential: advances in the development of animal models and future challenges. Arch. Toxicol., 84, 909-917.

Alizadeh, M., Ota, F., Hosoi, K., Kato, M., Sakai, T. and Satter, M.A. (2006): Altered allergic cytokine and antibody response in mice treated with Bisphenol A. J. Med. Invest., 53, 70-80.

Birmingham, N.P., Parvataneni, S., Hassan, H.M., Harkema, J., Samineni, S., Navuluri, L., Kelly, C.J. and Gangur, V. (2007): An adjuvant-free mouse model of tree nut allergy using hazelnut as a model tree nut. Int. Arch. Allergy Immunol., 144, 203-210.

Bowman, C.C. and Selgrade, M.K. (2008): Failure to induce oral tolerance in mice is predictive of dietary allergenic potency among foods with sensitizing capacity. Toxicol. Sci., 106, 435-443.

Broere, F., du Pré, M.F., van Berkel, L.A., Garssen, J., SchmidtWeber, C.B., Lambrecht, B.N., Hendriks, R.W., Nieuwenhuis, E.E.S., Kraal, G. and Samsom, J.N. (2009): Cyclooxygenase-2 in mucosal DC mediates induction of regulatory $\mathrm{T}$ cells in the intestine through suppression of IL-4. Mucosal Immunol., 2, 254-264.

Chmill, S., Kadow, S., Winter, M., Weighardt, H. and Esser, C. (2010): 2,3,7,8-Tetrachlorodibenzo-p-dioxin impairs stable establishment of oral tolerance in mice. Toxicol. Sci., 118, 98-107.

Clements, J.D., Hartzog, N.M. and Lyon, F.L. (1988): Adjuvant activity of Escherichia coli heat-labile enterotoxin and effect on the induction of oral tolerance in mice to unrelated protein antigens. Vaccine, 6, 269-277.

Diesner, S.C., Knittelfelder, R., Krishnamurthy, D., Pali-Schöll, I., Gajdzik, L., Jensen-Jarolim, E. and Untersmayr, E. (2008): Dose-dependent food allergy induction against ovalbumin under acid-suppression: a murine food allergy model. Immunol. Lett., 121, 45-51.

Elson, C.O. and Ealding, W. (1984): Cholera toxin feeding did not induce oral tolerance in mice and abrogated oral tolerance to an unrelated protein antigen. J. Immunol., 133, 2892-2897.

Finkelman, F.D. (2007): Anaphylaxis: Lessons from mouse models. J. Allergy Clin. Immunol., 120, 506-515.

Gaboriau-Routhiau, V. and Moreau, M.C. (1997): Oral tolerance to ovalbumin in mice: induction and long-term persistence unaffected by Staphylococcus aureus enterotoxin B and Clostridium perfringens type A enterotoxin. Pediatr. Res., 42, 503-508.

Ganeshan, K., Neilsen, C.V., Hadsaitong, A., Schleimer, R.P., Luo, X. and Bryce, P.J. (2009): Impairing oral tolerance promotes allergy and anaphylaxis: A new murine food allergy model. J. Allergy Clin. Immunol., 123, 231-238.

García, B.E., Gamboa, P.M., Asturias, J.A., López-Hoyos, M., 
Effective induction of oral anaphylaxis to ovalbumin in mice

Sanz,M.L., Caballero, M.T., García, J.M., Labrador, M., Lahoz, C., Longo Areso, N., Martínez Quesada, J., Mayorga, L. and Monteseirín, F.J. (2009): Clinical Immunology Committee; Spanish Society of Allergology and Clinical Immunology. Guidelines on the clinical usefulness of determination of specific immunoglobulin E to foods. Investig. Allergol. Clin. Immunol., 19, 423-32.

Gizzarelli, F., Corinti, S., Barletta, B., Iacovacci, P., Brunetto, B., Butteroni, C., Afferni, C., Onori, R., Miraglia, M., Panzini, G., Di Felice, G. and Tinghino, R. (2006): Evaluation of allergenicity of genetically modified soybean protein extract in a murine model of oral allergen-specific sensitization. Clin. Exp. Allergy, 36, 238-248.

Gonipeta, B., Parvataneni, S., Tempelman, R.J. and Gangur, V. (2009): An adjuvant-free mouse model to evaluate the allergenicity of milk whey protein. J. Dairy Sci., 92, 4738-4744.

Hsieh, K.Y., Hsu, C.I., Lin, J.Y., Tsai, C.C. and Lin, R.H. (2003): Oral administration of an edible-mushroom-derived protein inhibits the development of food-allergic reactions in mice. Clin. Exp. Allergy., 33, 1595-1602.

Kaneko, T., Terasawa, Y., Senoo, Y., Nagata, M. and Kuwata, T. (2000): Enhancing effect of dietary oil emulsions on immune responses to protein antigens fed to mice. Int .Arch. Allergy Immunol., 121, 317-323.

Kim, J.-H. and Ohsawa, M. (1995): Oral tolerance to ovalbumin in mice as a model for detecting modulators of the immunologic tolerance to a specific antigen. Biol. Pharm. Bull., 18, 854-858.

Ladics, G.S. (2008): Current codex guidelines for assessment of potential protein allergenicity. Food Chem. Toxicol., 46, Suppl. 10, S20-S23.

Lei, H.Y., Lee S.H. and Leir S.H. (1996): Antigen-induced anaphylactic death in mice. Int. Arch. Allergy Immunol., 109, 407-412.

Li, X.M., Schofield, B., Huang, C-K., Kleiner, G.I. and Sampson, H.A. (1999): A murine model of IgE-mediated cow's milk hypersensitivity. J. Allergy Clin. Immunol., 103, 206-214.

Li, X.M., Serebrisky, D., Lee, S.Y., Huang, C.K., Bardina, L., Schofield, B.H., Stanley, J.S., Burks, A.W., Bannon, G.A. and Sampson, H.A. (2000): A murine model of peanut anaphylaxis: $\mathrm{T}$ - and B-cell responses to a major peanut allergen mimic human responses. J. Allergy Clin. Immunol., 106, 150-158.

Louis, E., Franchimont, D., Deprez, M., Lamproye, A., Schaaf, N., Mahieu, P. and Belaiche, J. (1996): Decrease in systemic tolerance to fed ovalbumin in indomethacin-treated mice. Int. Arch. Allergy Immunol., 109, 21-26.

Matsuo, H., Morimoto, K., Akaki, T., Kaneko, S., Kuroda, T., Niihara, H., Hide, M. and Morita, E. (2005): Exercise and aspirin increase levels of circulating gliadin peptides in patients with wheat-dependent exercise-induced anaphylaxis. Clin. Exp. Allergy, 35, 461-466.

Miyajima, I., Dombrowicz, D., Martin, T.R. Ravetch, J.V., Kinet, J.P. and Gallii, S.J. (1997): Systemic anaphylaxis in the mouse can be mediated largely through $\mathrm{IgG} 1$ and $\mathrm{Fc}$ gamma RIII. Assessment of the cardiopulmonary changes, mast cell degranulation, and death associated with active or IgE- or IgG1-dependent passive anaphylaxis. J. Clin. Invest., 99, 901-914.

Miyake, Y., Tanaka, K., Sasaki, S. and Arakawa, M. (2011): Polyunsaturated fatty acid intake and prevalence of eczema and rhinoconjunctivitis in Japanese children: the Ryukyus Child Health Study. BMC Public Health., 11, 358.

Mota, I., Wong, D. and Sadun, E.H. (1968): Mouse homocytotropic antibodies. I. Specific differentiation between mouse $7 \mathrm{~S}$ gamma 1 and mouse reagin-like antibodies. Life Sci., 7, 1289-1293.
Mukai, K., Obata, K., Tsujimura, Y. and Karasuyama, H. (2009): New insights into the roles for basophils in acute and chronic allergy. Allergol. Int., 58, 11-19.

Nakamura, K., Inomata, N. and Ikezawa, Z. (2006): Dramatic augmentation of wheat allergy by aspirin in a dose-dependent manner. Ann. Allergy Asthma Immunol., 97, 712-713.

Oettgen, H.C., Martin, T.R., Wynshaw-Boris, A., Deng, C., Drazen, J.M. and Leder, P. (1994): Active anaphylaxis in IgE-deficient mice. Nature, 370, 367-370.

Patterson, J., Baxter, G., Lawrence J. and Duthie, G. (2006): Is there a role for dietary salicylates in health? Proc. Nutr. Soc., 65, 93-96.

Patterson, J.R., Gwendoline, B., Dreyer, J.S., Halket, J.M., Flynn, R. and Lawrence J.R. (2008): Salicylic acid sans aspirin in animals and man: Persistence in fasting and biosynthesis from benzoic acid. J. Agric. Food Chem., 56, 11648-11652.

Pecquet, S., Prioult, G., Campbell, J., German, B. and Turini, M. (2004): Commonly used drugs impair oral tolerance in mice. Ann. N.Y. Acad. Sci., 1029, 374-378.

Proust, B., Astier, C., Jacquenet, S., Ogier, V., Magueur, E., Roitel, O., Belcourt, C., Morisset, M., Moneret-Vautrin, D.A., Bihain, B.E. and Kanny, G. (2008): A single oral sensitization to peanut without adjuvant leads to anaphylaxis in mice. Int. Arch. Allergy Immunol., 146, 212-218.

Sala-Vila, A., Miles, E.A. and Calder, P.C. (2008): Fatty acid composition abnormalities in atopic disease: evidence explored and role in the disease process examined. Clin. Exp. Allergy, 38, 1432-1450.

Shirai, T., Matsui, T., Uto, T., Chida, K. and Nakamura, H. (2003): Nonsteroidal anti-inflammatory drugs enhance allergic reactions in a patient with wheat-induced anaphylaxis. Allergy, 58, 1071.

Schöll, I., Untersmayr, E., Bakos N., Roth-Walter F., Gleiss, A., Boltz-Nitulescu, G., Scheiner, O. and Jensen-Jarolim, E. (2005): Antiulcer drugs promote oral sensitization and hypersensitivity to hazelnut allergens in BALB/c mice and humans. Am. J. Clin. Nutr., 81 154-160.

Selgrade, M.K., Bowman, C.C., Ladics, G.S., Privalle, L. and Laessig, S.A. (2009): Safety assessment of biotechnology products for potential risk of food allergy: implications of new research. Toxicol. Sci., 110, 31-39.

Snider, D.P., Marshall, J.S., Perdue, M.H. and Liang, H. (1994): Production of IgE antibody and allergic sensitization of intestinal and peripheral tissues after oral immunization with protein $\mathrm{Ag}$ and cholera toxin. J. Immunol., 153, 647-657.

Strait, R.T., Morris S.C., Yang, M., Qu, X.-W. and Finkelman F.D. (2002): Pathways of anaphylaxis in the mouse. J. Allergy Clin. Immunol., 109, 658-668.

Strobel, S. and Mowat, A.M. (1998): Immune responses to dietary antigens: oral tolerance. Immunol. Today, 19, 173-181.

Tsujimura, Y., Obata, K., Mukai. K., Shindou, H., Yoshida, M., Nishikado, H., Kawano, Y., Minegishi, Y., Shimizu, T. and Karasuyama, H. (2008): Basophils play a pivotal role in immunoglobulin-G-mediated but not Immunoglobulin-E-mediated systemic anaphylaxis. Immunity, 28, 581-589.

Vadas, P., Gold, M., Perelman, B., Liss, G.M., Lack, G., Blyth, T., Simons, F.E., Simons, K.J., Cass, D. and Yeung, J. (2008) Platelet-activating factor, PAF acetylhydrolase, and severe anaphylaxis. N. Engl. J. Med., 358, 28-35.

van Wijk, F. Nierkens, S., Hassing, I., Feijen, M., Koppelman, S.J., de Jong, G.A.H., Pieters, R. and Knippels, L.M.J. (2005): The effect of the food matrix on in vivo immune responses to purified peanut allergens. Toxicol. Sci., 86, 333-341. 